\title{
Impact of three-dimensional polarization profiles on spin-dependent measurements in colliding beam experiments
}

\author{
Wolfram Fischer* and Alexander Bazilevsky \\ Brookhaven National Laboratory, Upton, New York 11973, USA \\ (Received 10 November 2011; published 2 April 2012)
}

\begin{abstract}
We derive the effect of 3-dimensional polarization profiles on the measured polarization in polarimeters, as well as the observed polarization and the polarization-weighted luminosity (figure of merit) in single and double spin measurements in colliding beam experiments. Applications to RHIC are discussed.
\end{abstract}

DOI: 10.1103/PhysRevSTAB.15.041001

PACS numbers: 29.20.db, 29.27.Hj

\section{INTRODUCTION}

The Relativistic Heavy Ion Collider (RHIC) is the only collider of spin polarized protons [1]. During beam acceleration and storage the polarization $P$ evolves. Polarization profiles, i.e., variation of polarization value versus betatron amplitudes, develop. These lead to profiles in the polarization measured in a polarimeter, i.e., variations of the polarization value versus the horizontal, vertical, or longitudinal space amplitude [2-4], and affect the observed polarization and polarization-weighted luminosity (figure of merit, FOM) in colliding beam experiments. The development of polarization profiles is the primary reason for the reduction of the average polarization $[4,5]$. We calculate average polarizations and figures of merit for profiles in all three dimensions, and give examples for RHIC. Like in RHIC we call the two colliding beams Blue and Yellow. We use the overbar to designate intensity-weighted averages as measured in polarimeters (e.g. $\bar{P}$ ), and angle brackets to designate luminosity-weighted averages in colliding beam experiments (e.g. $\langle P\rangle$ ).

\section{COORDINATES}

We use normalized horizontal, vertical, and longitudinal phase-space coordinates [6]

$$
\begin{gathered}
\left(x, \tilde{x}^{\prime}\right)=\left(x, \alpha_{x} x+\beta_{x} x^{\prime}\right) \quad\left(y, \tilde{y}^{\prime}\right)=\left(y, \alpha_{y} y+\beta_{y} y^{\prime}\right) \\
\left(s, \tilde{s}^{\prime}\right)=\left(\frac{\phi}{2 \pi} \frac{C}{h}, \frac{C \eta}{2 \pi Q_{s}} \frac{\delta p}{p}\right),
\end{gathered}
$$

where $\left(x, x^{\prime}\right)$ and $\left(y, y^{\prime}\right)$ are the horizontal and vertical phase-space coordinates, and $\left(\beta_{x}, \alpha_{x}\right)$ and $\left(\beta_{y}, \alpha_{y}\right)$ the respective lattice functions. $\phi$ is the rf phase, $C$ the circumference, $h$ the harmonic number, $\eta$ the slip factor, $Q_{s}$ the synchrotron tune, and $\delta p / p$ the relative momentum deviation. In the normalized coordinates the linear motion in

\footnotetext{
*Wolfram.Fischer@bnl.gov

Published by the American Physical Society under the terms of the Creative Commons Attribution 3.0 License. Further distribution of this work must maintain attribution to the author( $s)$ and the published article's title, journal citation, and DOI.
}

phase space is represented by a circle on a Poincaré surface of section, and all coordinates have the dimension length.

\section{DISTRIBUTIONS}

As was shown by measurements in RHIC transverse intensity profiles $[4,7,8]$ and transverse polarization profiles [2-4] can both be well approximated by Gaussian distributions. Similar approximations can also be used for longitudinal profiles $[9,10]$. The intensity distribution in phase space can then be written as

$$
\begin{aligned}
I\left(x, \tilde{x}^{\prime}, y, \tilde{y}^{\prime}, s, \tilde{s}^{\prime}\right)= & \frac{N_{b}}{(2 \pi)^{3} \sigma_{x}^{2} \sigma_{y}^{2} \sigma_{s}^{2}} \exp \left\{-\frac{x^{2}+\tilde{x}^{\prime 2}}{2 \sigma_{x}^{2}}\right. \\
& \left.-\frac{y^{2}+\tilde{y}^{\prime 2}}{2 \sigma_{y}^{2}}-\frac{s^{2}+\tilde{s}^{\prime 2}}{2 \sigma_{s}^{2}}\right\}
\end{aligned}
$$

where $N_{b}$ is the bunch intensity, and $\sigma_{x, y, s}$ are the rms beam sizes. The polarization $P$ can be approximated as

$$
P\left(x, \tilde{x}^{\prime}, y, \tilde{y}^{\prime}, s, \tilde{s}^{\prime}\right)=P_{0} \exp \left\{-\frac{x^{2}+\tilde{x}^{\prime 2}}{2 \sigma_{x, P}^{2}}-\frac{y^{2}+\tilde{y}^{\prime 2}}{2 \sigma_{y, P}^{2}}-\frac{s^{2}+\tilde{s}^{\prime 2}}{2 \sigma_{s, P}^{2}}\right\} .
$$

With this dependence the polarization is a function of the normalized horizontal $\left(\sqrt{x^{2}+\tilde{x}^{\prime 2}}\right)$, vertical $\left(\sqrt{y^{2}+\tilde{y}^{\prime 2}}\right)$, and longitudinal $\left(\sqrt{s^{2}+\tilde{s}^{12}}\right)$ betatron amplitudes. The maximum polarization $P_{0}$ is reached for zero amplitudes in all dimensions. We also introduce the quantities

$$
R_{x}:=\frac{\sigma_{x}^{2}}{\sigma_{x, P}^{2}}, \quad R_{y}:=\frac{\sigma_{y}^{2}}{\sigma_{y, P}^{2}}, \quad \text { and } \quad R_{s}:=\frac{\sigma_{s}^{2}}{\sigma_{s, P}^{2}}
$$

that parametrize the polarization profile, and with which Eq. (3) can be written as

$$
\begin{aligned}
P\left(x, \tilde{x}^{\prime}, y, \tilde{y}^{\prime}, s, \tilde{s}^{\prime}\right)= & P_{0} \exp \left\{-R_{x} \frac{x^{2}+\tilde{x}^{\prime 2}}{2 \sigma_{x}^{2}}\right. \\
& \left.-R_{y} \frac{y^{2}+\tilde{y}^{\prime 2}}{2 \sigma_{y}^{2}}-R_{s} \frac{s^{2}+\tilde{s}^{2}}{2 \sigma_{s}^{2}}\right\} .
\end{aligned}
$$


Without any polarization profiles we have $\sigma_{x, P} \rightarrow \infty$, $\sigma_{y, P} \rightarrow \infty, \sigma_{s, P} \rightarrow \infty$, and $R_{x}=R_{y}=R_{s}=0$.

In general, the phase-space distributions $I$ [Eq. (2)] and $P$ [Eq. (5)] are dependent on the location in a storage ring. For small enough betatron amplitudes the betatron motion can be linearized around the closed orbit and its representation on the Poincare surface of sections is a circle at all locations in the ring. In this case, the distribution $I$ is independent of the ring location. The situation is more complicated for polarization profiles $P$ although it is still a reasonable assumption that the profile parameters $R_{x, y, s}$ are independent of the ring location for small betatron amplitudes [6,11].

\section{POLARIZATION MEASUREMENTS}

Two types of polarimeters are used in RHIC to provide proton beam polarization measurements. One type of polarimeter uses a polarized atomic hydrogen gas target $[12,13]$ and measures the average polarization over all particles:

$$
\begin{aligned}
\bar{P} & =\frac{\int_{-\infty}^{+\infty} \cdots \int d x d \tilde{x}^{\prime} d y d \tilde{y}^{\prime} d s d \tilde{s}^{\prime} P(x, \ldots) I(x, \ldots)}{\int_{-\infty}^{+\infty} \cdots \int d x d \tilde{x}^{\prime} d y d \tilde{y}^{\prime} d s d \tilde{s}^{\prime} I(x, \ldots)} \\
& =\frac{P_{0}}{\left(1+R_{x}\right)\left(1+R_{y}\right)\left(1+R_{s}\right)} .
\end{aligned}
$$

Here and in the following, the overbar denotes the intensity-weighted average. The other type of polarimeter uses an ultrathin carbon target [14-16] and is capable of measuring intensity and polarization profiles in both transverse directions. In a horizontal profile measurement with a thin vertical target, we have

$$
\begin{aligned}
\overline{P_{x}}(x) & =\frac{\int_{-\infty}^{+\infty} \cdots \int d \tilde{x}^{\prime} d y d \tilde{y}^{\prime} d s d \tilde{s}^{\prime} P(x, \ldots) I(x, \ldots)}{\int_{-\infty}^{+\infty} \cdots \int d \tilde{x}^{\prime} d y d \tilde{y}^{\prime} d s d \tilde{s}^{\prime} I(x, \ldots)} \\
& =\frac{P_{0}}{\sqrt{1+R_{x}}\left(1+R_{y}\right)\left(1+R_{s}\right)} \exp \left\{-\frac{R_{x} x^{2}}{2 \sigma_{x}^{2}}\right\} .
\end{aligned}
$$

Similarly, we have for a vertical profile measurement with a thin horizontal target

$$
\overline{P_{y}}(y)=\frac{P_{0}}{\left(1+R_{x}\right) \sqrt{1+R_{y}}\left(1+R_{s}\right)} \exp \left\{-\frac{R_{y} y^{2}}{2 \sigma_{y}^{2}}\right\},
$$

and for a longitudinal profile measurement

$$
\overline{P_{s}}(s)=\frac{P_{0}}{\left(1+R_{x}\right)\left(1+R_{y}\right) \sqrt{1+R_{s}}} \exp \left\{-\frac{R_{s} s^{2}}{2 \sigma_{s}^{2}}\right\} .
$$

A longitudinal profile can be obtained through time binning in polarimeter measurements [10].

\section{LUMINOSITY}

For the following we recall the luminosity formula $[6,17,18]$

$$
\begin{aligned}
\mathcal{L}= & f_{c} \int_{-\infty}^{+\infty} \cdots \int d x d y d s d t \hat{I}_{B}(x, \ldots, t) \hat{I}_{Y}(x, \ldots, t) \\
& \times t \sqrt{\left(\vec{v}_{B}-\vec{v}_{Y}\right)^{2}-\frac{\left(\vec{v}_{B} \times \vec{v}_{Y}\right)^{2}}{c^{2}}},
\end{aligned}
$$

where $f_{c}$ is the bunch collision frequency, and the subscripts $B$ and $Y$ describe quantities of the Blue and Yellow beams, respectively. Note that the distributions $\hat{I}$ are only 3-dimensional and also time dependent:

$$
\begin{aligned}
\hat{I}(x, y, s, t) & =\int_{-\infty}^{+\infty} \cdots \int d \tilde{x}^{\prime} d \tilde{y}^{\prime} d \tilde{s}^{\prime} I(x, \ldots, t) \\
& =\frac{N_{b}}{(2 \pi)^{3 / 2} \sigma_{x} \sigma_{y} \sigma_{s}} \exp \left\{-\frac{x^{2}}{2 \sigma_{x}^{2}}-\frac{y^{2}}{2 \sigma_{y}^{2}}-\frac{s^{2}}{2 \sigma_{s}^{2}}\right\} .
\end{aligned}
$$

$\vec{v}$ is the common velocity of particles in the bunch, and $c$ the speed of light. The rms beam sizes $\sigma_{x, y, s}$ are functions of the time $t$. With neither transverse offset nor crossing angle, the luminosity can be written as [17,19]

$$
\mathcal{L}=\frac{f_{c} N_{b, B} N_{b, Y}}{2 \pi \sqrt{\left(\sigma_{x, B}^{* 2}+\sigma_{x, Y}^{* 2}\right)\left(\sigma_{y, B}^{* 2}+\sigma_{y, Y}^{* 2}\right)}} h\left(t_{x}, t_{y}\right),
$$

where the superscript $*$ denotes quantities at the interaction point, and the function $h\left(t_{x}, t_{y}\right)$ is the hourglass factor

$$
h\left(t_{x}, t_{y}\right)=\int_{-\infty}^{\infty} \frac{d t}{\sqrt{\pi}} \frac{\exp \left(-t^{2}\right)}{\sqrt{\left(1+t^{2} / t_{x}^{2}\right)\left(1+t^{2} / t_{y}^{2}\right)}}
$$

with

$$
t_{x}^{2}=\frac{2\left(\sigma_{x, B}^{* 2}+\sigma_{x, Y}^{* 2}\right)}{\left(\sigma_{s, B}^{2}+\sigma_{s, Y}^{2}\right)\left(\sigma_{x, B}^{* 2} / \beta_{x, B}^{* 2}+\sigma_{x, Y}^{* 2} / \beta_{x, Y}^{* 2}\right)} .
$$

A similar expression holds for $t_{y}^{2}$.

\section{AVERAGE POLARIZATIONS AND FIGURES OF MERIT IN COLLIDING BEAM EXPERIMENTS}

Let us introduce polarization moments for two colliding beams as

$$
M_{m n}=\left\langle P_{B}^{m} \cdot P_{Y}^{n}\right\rangle,
$$

where $m$ and $n$ are non-negative integers and the angle brackets indicate the luminosity-weighted average over the polarization distributions. $P_{B}$ and $P_{Y}$ denote the polarizations of the Blue and Yellow beam, respectively.

Important quantities for a collider experiment are average polarizations and figures of merit. For single spin measurements with the Blue and Yellow beams, respectively, these can be expressed through the polarization moments $M_{m n}$ as

$$
\left\langle P_{B}\right\rangle \equiv M_{10} \quad \mathrm{FOM}_{B} \equiv \mathcal{L}\left\langle P_{B}^{2}\right\rangle=\mathcal{L} M_{20}
$$

and 


$$
\left\langle P_{Y}\right\rangle \equiv M_{01} \quad \mathrm{FOM}_{Y} \equiv \mathcal{L}\left\langle P_{Y}^{2}\right\rangle=\mathcal{L} M_{02} .
$$

For double spin experiments we have

$$
\left\langle P_{B} \cdot P_{Y}\right\rangle \equiv M_{11} \quad \operatorname{FOM}_{B Y} \equiv \mathcal{L}\left\langle P_{B}^{2} \cdot P_{Y}^{2}\right\rangle=\mathcal{L} M_{22} .
$$

Polarization profiles dilute the measured spin asymmetries and rescaling is needed to get the physics asymmetries. Statistical uncertainties in the measurement scale as $1 / \sqrt{\mathrm{FOM}}$, so figures of merit describe the experimental sensitivity or resolution.

We now calculate the moments $M_{m n}$ using the luminosity formulas in the previous section. With Eq. (15) we have

$$
\begin{aligned}
M_{m n}= & \frac{f_{c}}{\mathcal{L}} \int_{-\infty}^{+\infty} \cdots \int d x d y d s d t \hat{I}_{B}(x, \ldots, t) \\
& \times \hat{I}_{Y}(x, \ldots, t) \hat{P}_{B}^{m}(x, \ldots, t) \hat{P}_{Y}^{n}(x, \ldots, t) \\
& \times \sqrt{\left(\vec{v}_{B}-\vec{v}_{Y}\right)^{2}-\frac{\left(\vec{v}_{B} \times \vec{v}_{Y}\right)^{2}}{c^{2}}},
\end{aligned}
$$

where the time-dependent polarization functions in 3 spacial dimensions $\hat{P}^{k}(k=m, n)$ are given by

$$
\begin{aligned}
\hat{P}^{k}(x, y, s, t)= & \frac{\int_{-\infty}^{+\infty} \cdots \int d \tilde{x}^{\prime} d \tilde{y}^{\prime} d \tilde{s}^{\prime} P^{k}(x, \ldots, t) I(x, \ldots, t)}{\int_{-\infty}^{+\infty} \cdots \int d \tilde{x}^{\prime} d \tilde{y}^{\prime} d \tilde{s}^{\prime} I(x, \ldots, t)} \\
= & \frac{P_{0}^{k}}{\sqrt{\left(1+k R_{x}\right)\left(1+k R_{y}\right)\left(1+k R_{s}\right)}} \\
& \times \exp \left\{-\frac{k R_{x} x^{2}}{2 \sigma_{x}^{2}}-\frac{k R_{y} y^{2}}{2 \sigma_{y}^{2}}-\frac{k R_{s} s^{2}}{2 \sigma_{s}^{2}}\right\} .
\end{aligned}
$$

Equation (19) can be expressed as

$$
\begin{aligned}
M_{m n}= & \frac{P_{0, B}^{m} P_{0, Y}^{n}}{\mathcal{L}} \prod_{i=x, y, s} \frac{1}{\left(1+m R_{i, B}\right)\left(1+n R_{i, Y}\right)} \\
& \times f_{c} \int_{-\infty}^{+\infty} \cdots \int d x d y d s d t \frac{N_{b, B} N_{b, Y}}{(2 \pi)^{3}} \\
& \times \prod_{i=x, y, s} \frac{\sqrt{\left(1+m R_{i, B}\right)\left(1+n R_{i, Y}\right)}}{\sigma_{i, B} \sigma_{i, Y}} \\
& \times \prod_{i=x, y, s} \exp \left\{-\frac{\left(1+m R_{i, B}\right) i^{2}}{2 \sigma_{i, B}^{2}}-\frac{\left(1+n R_{i, Y}\right) i^{2}}{2 \sigma_{i, Y}^{2}}\right\} \\
& \times \sqrt{\left(\vec{v}_{B}-\vec{v}_{Y}\right)^{2}-\frac{\left(\vec{v}_{B} \times \vec{v}_{Y}\right)^{2}}{c^{2}}} .
\end{aligned}
$$

The last 4 lines of the above expression have the same form as Eq. (10). The solution of Eq. (12) can be used with the replacements

$$
\sigma_{i, B}^{* 2} \rightarrow \sigma_{i, B}^{* 2} /\left(1+m R_{i, B}\right) \quad \sigma_{i, Y}^{* 2} \rightarrow \sigma_{i, Y}^{* 2} /\left(1+n R_{i, Y}\right)
$$

for $i=x, y$ and

$$
\sigma_{s, B}^{2} \rightarrow \sigma_{s, B}^{2} /\left(1+m R_{s, B}\right) \quad \sigma_{s, Y}^{2} \rightarrow \sigma_{s, Y}^{2} /\left(1+n R_{s, Y}\right) .
$$

We obtain

$$
\begin{aligned}
M_{m n}= & P_{0, B}^{m} P_{0, Y}^{n} \prod_{i=x, y, S} \frac{1}{\left(1+m R_{i, B}\right)\left(1+n R_{i, Y}\right)} \\
& \times \frac{\sqrt{\left(\sigma_{x, B}^{* 2}+\sigma_{x, Y}^{* 2}\right)\left(\sigma_{y, B}^{* 2}+\sigma_{y, Y}^{* 2}\right)}}{\sqrt{\left(\frac{\sigma_{x, B}^{* 2}}{1+m R_{x, B}}+\frac{\sigma_{x, Y}^{* 2}}{1+n R_{x, Y}}\right)\left(\frac{\sigma_{y, B}^{* 2}}{1+m R_{y, B}}+\frac{\sigma_{y, Y}^{* 2}}{1+n R_{y, Y}}\right)}} \frac{h\left(t_{x, m n}, t_{y, m n}\right)}{h\left(t_{x}, t_{y}\right)}
\end{aligned}
$$

with

$$
t_{x, m n}^{2}=\frac{2}{\left(\frac{\sigma_{s, B}^{2}}{1+m R_{s, B}}+\frac{\sigma_{s, Y}^{2}}{1+n R_{s, Y}}\right)} \frac{\left(\frac{\sigma_{x, B}^{* 2}}{1+m R_{x, B}}+\frac{\sigma_{x, Y}^{* 2}}{1+n R_{x, Y}}\right)}{\left(\frac{\sigma_{x, B}^{* 2}}{\left(1+m R_{x, B}\right) \beta_{x, B}^{* 2}}+\frac{\sigma_{x, Y}^{* 2}}{\left(1+n R_{x, Y}\right) \beta_{x, Y}^{* 2}}\right)}
$$

and a similar expression for $t_{y, m n}^{2}$.

Note that due to polarization profiles, the polarizations observed by colliding beam experiments [Eqs. (16) and (17) with the solution of Eq. (24)] generally differ from the average polarization measured by a polarimeter [Eq. (6)].

\section{SIMPLIFIED CASE}

To simplify the general solution of Eq. (24) considerably, we make the following assumptions: (i) short bunches, i.e., no hourglass effect- $\sigma_{s, B}, \sigma_{s, Y} \ll \beta_{x, B}^{*}$, $\beta_{y, B}^{*}, \beta_{x, Y}^{*}, \beta_{y, Y}^{*} \Rightarrow h\left(t_{x}, t_{y}\right)=1$; (ii) no longitudinal polarization profile $-R_{s, B}=R_{s, Y}=0 \Rightarrow h\left(t_{x, m n}, t_{y, m n}\right)=1$; (iii) equal transverse polarization profiles in both beams $-P_{0, B}=P_{0, Y}=P_{0}, \quad R_{x, B}=R_{x, Y}=R_{x}, \quad R_{y, B}=$ $R_{y, Y}=R_{y}$; (iv) round beams of the same size in both rings- $\sigma_{x, B}^{*}=\sigma_{y, B}^{*}=\sigma_{x, Y}^{*}=\sigma_{y, Y}^{*}=\sigma^{*}$. Equation (24) can then be written as

$$
\begin{aligned}
M_{m n}= & \frac{P_{0}^{m+n}}{\sqrt{\left(1+\frac{m+n}{2} R_{x}\right)\left(1+\frac{m+n}{2} R_{y}\right)}} \\
& \times \frac{1}{\sqrt{\left(1+m R_{x}\right)\left(1+n R_{x}\right)\left(1+m R_{y}\right)\left(1+n R_{y}\right)}}
\end{aligned}
$$

and the cases of Eqs. (16)-(18) become

$$
\begin{aligned}
\left\langle P_{B}\right\rangle \quad \text { or }\left\langle P_{Y}\right\rangle= & \frac{P_{0}}{\left[\left(1+\frac{1}{2} R_{x}\right)\left(1+\frac{1}{2} R_{y}\right)\right]^{1 / 2}} \\
& \times \frac{1}{\left[\left(1+R_{x}\right)\left(1+R_{y}\right)\right]^{1 / 2}}, \\
\left\langle P_{B}^{2}\right\rangle \quad \text { or }\left\langle P_{Y}^{2}\right\rangle= & \frac{P_{0}^{2}}{\left[\left(1+R_{x}\right)\left(1+R_{Y}\right)\right]^{1 / 2}} \\
& \times \frac{1}{\left[\left(1+2 R_{x}\right)\left(1+2 R_{y}\right)\right]^{1 / 2}},
\end{aligned}
$$




$$
\begin{gathered}
\left\langle P_{B} \cdot P_{Y}\right\rangle=\frac{P_{0}^{2}}{\left[\left(1+R_{x}\right)\left(1+R_{y}\right)\right]^{3 / 2}}, \\
\left\langle P_{B}^{2} \cdot P_{Y}^{2}\right\rangle=\frac{P_{0}^{4}}{\left[\left(1+2 R_{x}\right)\left(1+2 R_{y}\right)\right]^{3 / 2}} .
\end{gathered}
$$

The ratio between the polarization $\bar{P}$ measured by a polarimeter and the polarization $\langle P\rangle$ observed in a single spin colliding beam experiment is for the simplified case

$$
\begin{aligned}
\frac{\overline{P_{B}}}{\left\langle P_{B}\right\rangle} \text { or } \frac{\overline{P_{Y}}}{\left\langle P_{Y}\right\rangle} & =\sqrt{\frac{\left(1+\frac{1}{2} R_{x}\right)\left(1+\frac{1}{2} R_{y}\right)}{\left(1+R_{x}\right)\left(1+R_{y}\right)}} \\
& \approx 1-\frac{R_{x}+R_{y}}{4} \quad \text { for } R_{x}, R_{y} \ll 1 .
\end{aligned}
$$

\section{EXAMPLE FROM RHIC}

For illustration we take the RHIC $250 \mathrm{GeV}$ polarized proton run in 2011 [9], with $\beta_{x, y}^{*}=0.6 \mathrm{~m}$ [20] at Interaction Point 8 (PHENIX experiment) and $\sigma_{s}=$ $0.6 \mathrm{~m}$ [9] in both beams.

We consider the two cases of $R_{x}=0$ and $R_{x}=R_{y}$, both with equal polarization profiles in both beams. The former
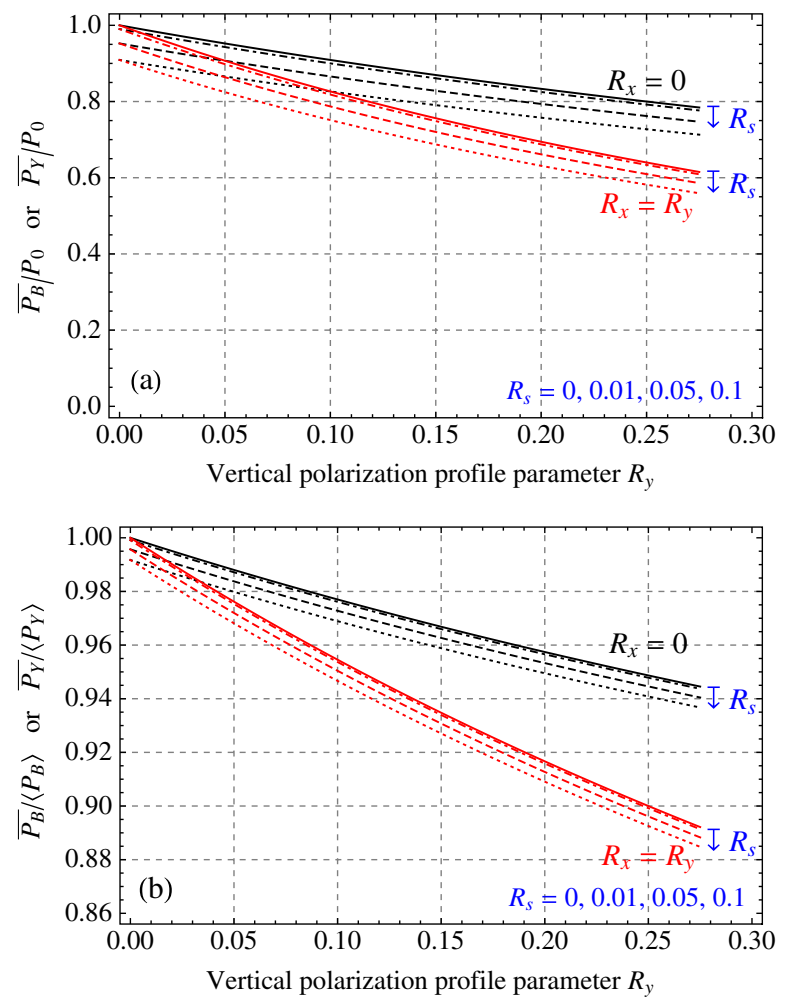

FIG. 1. (a) Relative reduction of the average polarization, $\overline{P_{B}} / P_{0}$ or $\overline{P_{Y}} / P_{0}$, as a function of the vertical profile parameter $R_{y}$. (b) Ratio of the polarization measured by a polarimeter to the average polarization in single spin colliding beam experiments, $\overline{P_{B}} /\left\langle P_{B}\right\rangle$ or $\overline{P_{Y}} /\left\langle P_{Y}\right\rangle$. The cases $R_{x}=0$ and $R_{x}=R_{y}$ are shown for $R_{S}=0,0.01,0.05,0.1$ each. case is expected if all machines in the acceleration chain are perfectly flat, the spin direction is always vertical, and the horizontal and vertical planes decoupled. The latter case is expected for fully coupled machines. Profile parameters of $R_{x} \approx R_{y} \approx 0.2$ were observed with an ultrathin carbon target at $250 \mathrm{GeV}$ in 2011 [4]. The measurements of longitudinal profile parameter $R_{s}$ have not been yet finalized but were found to be small [10].

Figure 1(a) shows the relative reduction (i.e. relative to the case without polarization profiles) of the average polarization $\bar{P}$ measured by a polarimeter as a function of the vertical profile parameter $R_{y}$. The two cases $R_{x}=0$ and $R_{x}=R_{y}$ are shown for $R_{s}=0,0.01,0.05,0.1$ each. Figure 1(b) displays the ratio of the polarization measured by a polarimeter to the polarization seen in a single spin colliding beam experiment.

Figure 2 exhibits the effect of the polarization profiles on the average polarization and figure of merit in single spin colliding beam experiments. Figure 3 shows the effect in double spin experiments.

The evaluations above assume that the beam polarization profiles (namely the profile parameters $R_{x, y, s}$ ) do not change from the polarimeter location in the ring to the experimental collision points, even if they are separated by
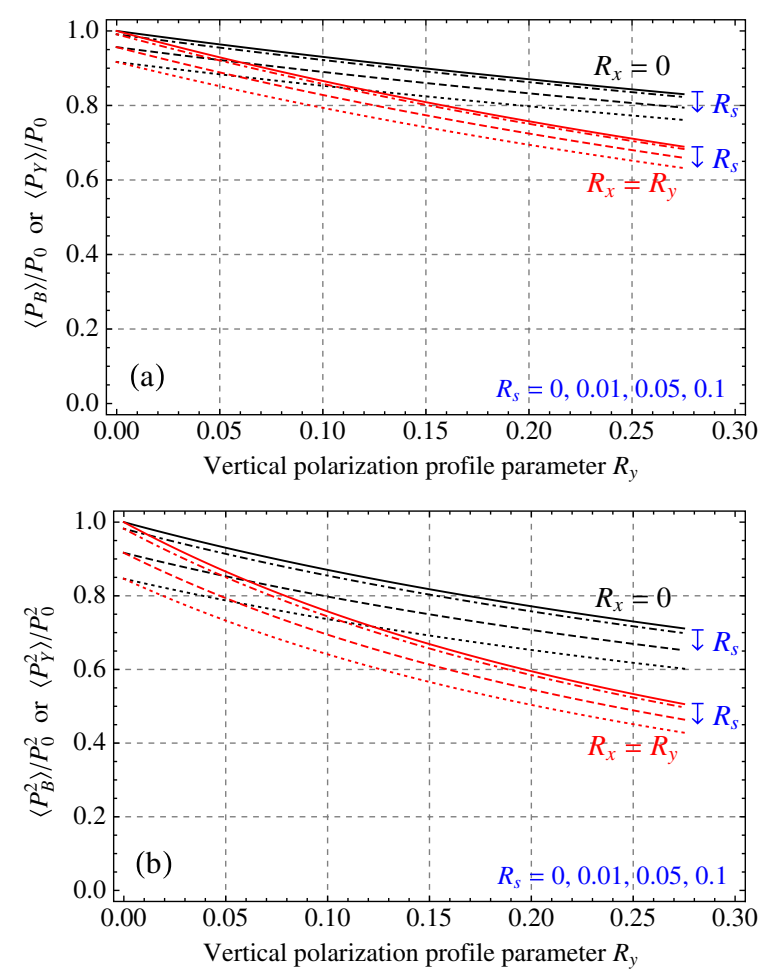

FIG. 2. For single spin colliding beam experiments (a) relative reduction of the average polarization $\left\langle P_{B}\right\rangle / P_{0}$ or $\left\langle P_{Y}\right\rangle / P_{0}$, and (b) relative reduction of the figure of merit $\left\langle P_{B}^{2}\right\rangle / P_{0}^{2}$ or $\left\langle P_{Y}^{2}\right\rangle / P_{0}^{2}$, as a function of the vertical profile parameter $R_{y}$. The cases $R_{x}=0$ and $R_{x}=R_{y}$ are shown for $R_{s}=0,0.01,0.05,0.1$ each. 

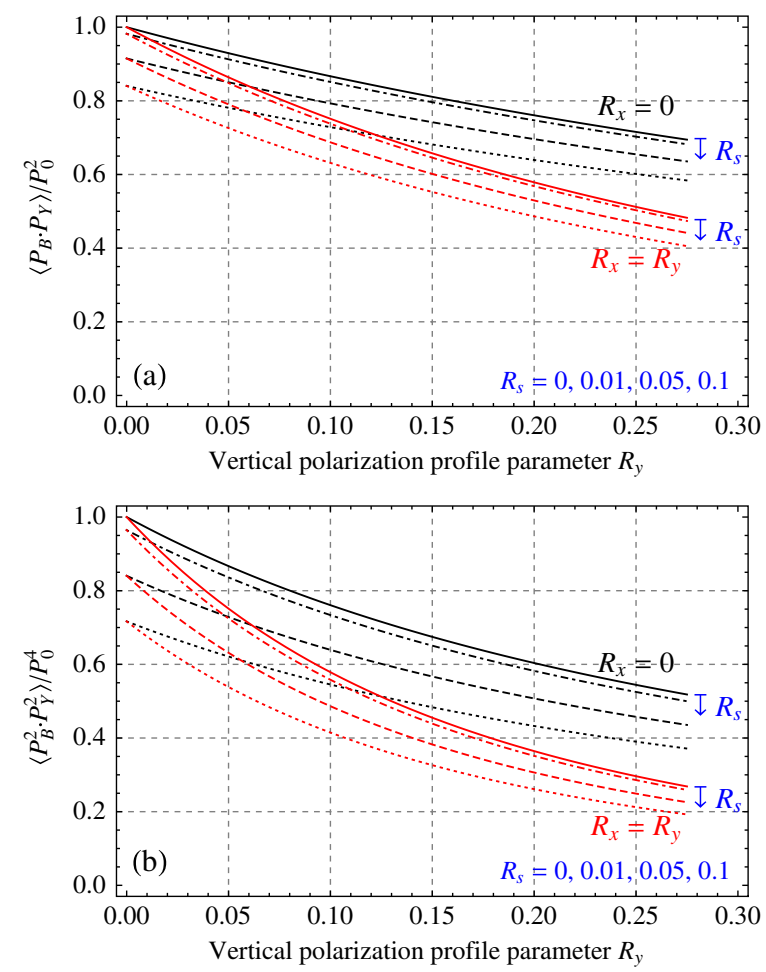

FIG. 3. For double spin colliding beam experiments (a) relative reduction of the average polarization function $\left\langle P_{B} \cdot P_{Y}\right\rangle / P_{0}^{2}$, and (b) relative reduction of the figure of merit $\left\langle P_{B}^{2} \cdot P_{Y}^{2}\right\rangle / P_{0}^{4}$, as a function of the vertical profile parameter $R_{y}$. Assuming the same polarization profiles in both beams the cases $R_{x}=0$ and $R_{x}=R_{y}$ are shown for $R_{s}=0,0.01,0.05,0.1$ each.

"Siberian snakes" (magnets that flip the spin direction by 180 degrees), and by spin rotators (magnets that rotate the spin from vertical to longitudinal or horizontal, and back) [1]. This is a separate topic for careful investigation and confirmation. A dedicated simulation study is under way to better understand proton spin dynamics in RHIC, and in particular to test these assumptions [21].

\section{SUMMARY}

The development of polarization profiles have an impact on both the average beam polarization and the polarization-weighted luminosity (figures of merit) in colliding beam experiments. An example is RHIC with typical profile parameter $R$ values of 0.2 in both transverse planes. Because of the profiles, the polarization measured by polarimeters is different from the polarization observed by the experiments, and corrections depend on profiles in all three planes. For precision spin experiments polarimetry must provide measurements and monitoring of the polarization profiles in all three dimensions. We analytically derived formulas to quantify these effects for 3-dimensional Gaussian profiles, which is a valid assumption for RHIC.

\section{ACKNOWLEDGMENTS}

The authors are thankful for discussions with the members of the RHIC Spin Collaboration, in particular E. Aschenauer, M. Bai, M. Blaskiewicz, H. Huang, W. W. MacKay, A. Poblaguev, T. Roser, V. Ranjbar, B. Schmidke, D. Smirnov, and A. Zelenski. This work was supported by Brookhaven Science Associates, LLC under Contract No. DE-AC02-98CH10886 with the U.S. Department of Energy.

[1] T. Roser, W. W. MacKay et al., "Configuration manual, polarized proton collider at RHIC," Rev. 2 (2006).

[2] A. Bazilevsky, in The XIIth International Workshop on Polarized Sources, Targets, and Polarimetry PSPT2007, New York, USA (2007) [http://www.c-ad.bnl.gov/ pst2007/program.html].

[3] A. Bazilevsky, I. Alekseev, E. Aschenauer, G. Atoyan, A. Bravar, G. Bunce, G. Boyle, C.M. Camacho, V. Dharmawardane, R. Gill, H. Huang, S. Lee, X. Li, H. Liu, Y. Makdisi, B. Morozov, I. Nakagawa, H. Okada, D. Svirida, M. Sievertz, and A. Zelenski, in The XIIIth Advanced Research Workshop on High Energy Spin Physics, DSPIN-09, Dubna, Russia (2009), pp. 389-396.

[4] A. Zelenski, in The XIVth International Workshop on Polarized Sources, Targets, and Polarimetry PSPT2011, St. Petersburg, Russia (2011).

[5] H. Huang, L. Ahrens, I. G. Alekseev, E. Aschenauer, G. Atoian, M. Bai, A. Bazilevsky, M. Blaskiewicz, J. M. Brennan, K. A. Brown, D. Bruno, R. Connolly, A. Dion, T. D'Ottavio, K. A. Drees, W. Fischer, C. J. Gardner, J. W. Glenn, X. Gu, M. Harvey, T. Hayes, L. Hoff, R. L. Hulsart, J. S. Laster, C. Liu, Y. Luo, W. W. MacKay, Y. Makdisi, G. J. Marr, A. Marusic, F. Meot, K. Mernick, R. J. Michnoff, M. G. Minty, C. Montag, J. Morris, S. Nemesure, A. Poblaguev, V. Ptitsyn, V. Ranjbar, G. Robert-Demolaize, T. Roser, B. Schmidtke, V. Schoefer, F. Severino, D. Smirnov, K. Smith, D. Steski, D. Svirida, S. Tepikian, D. Trbojevic, N. Tsoupas, J. E. Tuozzolo, G. Wang, M. Wilinski, K. Yip, A. Zaltsman, A. Zelenski, K. Zeno, and S. Y. Zhang, in Proceedings International Particle Accelerator Conference 2011, San Sebastian, Spain (EPS-AG, 2011), pp. 1888-1890 [http://epaper .kek.jp/IPAC2011/index.htm].

[6] M. Conte and W. W. MacKay, An introduction to the Physics of Particle Accelerators (World Scientific, Singapore, 2008), 2nd ed.

[7] A. Zelenski, G. Atoyan, G. Bunce, A. Bazilevsky, R. Connolly, D. Gassner, R. Gill, H. Huang, G. Mahler, Y. Makdisi, B. Morozov, S. Nemesure, T. Russo, D. Steski, M. Sivertz, S. Tepikian, R. Todd, and K. Yip, in Proceedings of the 23rd Particle Accelerator Conference, Vancouver, Canada, 2009 (IEEE, Piscataway, NJ, 2009), pp. 3488-3490.

[8] R. Connolly, J. Fite, S. Jao, S. Tepikian, and C. Trabocci, in Proceedings of the 14th Beam Instrumentation Workshop, Santa Fe, New Mexico (LANL, Santa Fe, 2010), pp. 116-118. 
[9] H. Huang, L. A. Ahrens, E. C. Aschenauer, G. Atoian, M. Bai, A. Bazilevsky, J. Beebe-Wang, M. Blaskiewicz, J. M. Brennan, K. A. Brown, D. Bruno, R. Connolly, T. D’Ottavio, A. Dion, K. A. Drees, W. Fischer, C. J. Gardner, J. W. Glenn, X. Gu, M. Harvey, T. Hayes, L. T. Hoff, R. L. Hulsart, J. S. Laster, C. Liu, Y. Luo, W. W. MacKay, Y. Makdisi, M. Mapes, G. J. Marr, A. Marusic, F. Méot, K. Mernick, R. J. Michnoff, M. G. Minty, C. Montag, J. Morris, S. Nemesure, A. Poblaguev, V. Ptitsyn, V.H. Ranjbar, G. Robert-Demolaize, T. Roser, W. B. Schmidke, V. Schoefer, F. Severino, D. Smirnov, K. S. Smith, D. Steski, S. Tepikian, D. Trbojevic, N. Tsoupas, J. E. Tuozzolo, G. Wang, M. Wilinski, K. Yip, A. Zaltsman, A. Zelenski, K. Zeno, and S. Y. Zhang, Proceedings of the 2011 Particle Accelerator Conference, New York, USA (IEEE, New York, 2011), pp. 41-45.

[10] RHIC polarimetry group, (private communication).

[11] G. H. Hoffstaetter, High-Energy Polarized Proton Beams-A Modern View, Springer Tracts in Modern Physics Vol. 218 (Springer, New York, 2006).

[12] A. Zelenski et al., in Proceedings SPIN 2002, Upton, NY, USA, AIP Conf. Proc. No. 675 (AIP, New York, 2002), pp. 954-962.

[13] T. Wise et al., in Proceedings SPIN 2004, Trieste, Italy (World Scientific, Singapore, 2004), pp. 757-760.

[14] H. Huang, M. Bai, G. Bunce, Y. Makdisi, T. Roser, K. Imai, M. Nakamura, J. Tojo, K. Yamamoto, L. Zhu, B.
Bassalleck, S. Eilerts, D. E. Fields, B. Lewis, B. Smith, T. L. Thomas, D. Wolfe, Y. Yoto, N. Hayoshi, M. Ishihara, K. Kurita, M. Okamura, N. Saito, A. Taketani, D. Underwood, J. Doskow, K. Kwiatkowski, B. Lozowski, H. O. Meyer, B. v. Przewoski, T. Rinckel, S. B. Nurushev, M. N. Strikhanov, M.F. Runtzo, I. G. Alekseev, D. N. Svirida, A. Deshpande, and V. Hughes, in Proceedings Particle Accelerator Conference 1999, New York, USA (IEEE, New York, 1999), pp. 471-473.

[15] H. Huang and K. Kurita, Beam Instrumentation Workshop 2006 (BIW06), Batavia, IL, USA, AIP Conf. Proc. No. 868 (AIP, New York, 2006), pp. 3-21.

[16] A. Bazilevsky, A. Bravar, G. Bunce, R. Gill, H. Huang, Y. Makdisi, I. Nakagawa, B. Morozov, D. Steski, M. Sivertz, K. Yip, A. Zelenski, V. Dharmawardane, I. Alekseev, D. Svirida, and W. Lozowski, in Proceedings of the European Particle Accelerator Conference 2008, Genoa, Italy (EPS-AG, Genoa, Italy, 2008), pp. 11401142.

[17] M.A. Furman and M.S. Zisman, in Handbook of Accelerator Physics and Engineering, edited by A.W. Chao and M. Tigner (World Scientific, Singapore, 1999).

[18] W. W. MacKay, Report No. BNL C-A/AP/89, 2002.

[19] M.A. Furman, in Proceedings Particle Accelerator Conference 1991, San Francisco, USA (IEEE, New York, 1991), pp. 422-424.

[20] M. Bai (private communication).

[21] V. Ranjbar (private communication). 